\title{
HIGH-FIDELITY EX-CORE CAPABILITIES IN VERA
}

\author{
Tara Pandya, Katherine Royston, Eva Davidson, Tom Evans, Andrew Godfrey, \\ Shane Henderson, Cole Gentry, Shane Stimpson, and Benjamin Collins ${ }^{1}$ * \\ ${ }^{1}$ Oak Ridge National Laboratory \\ P.O. Box 5800, Oak Ridge, TN 37831-6170
}

pandyatm@ornl.gov, kr4@ornl.gov, e32@ornl.gov, 9te@ornl.gov, agm@ornl.gov, oxh@ornl.gov,.cgd@ornl.gov,ggs@ornl.gov,.bn7@ornl.gov

\begin{abstract}
High-fidelity nuclear reactor calculations have become increasingly important when considering lifetime extensions of the current nuclear fleet. The need for performing large calculations has led to development of advanced, novel methods for faster and more efficient computing system use. Under the Consortium for Advanced Simulation of Light Water Reactors (CASL), we have enabled the capability to perform high-fidelity ex-core calculations in the Virtual Environment for Reactor Applications (VERA) by coupling with the Shift Monte Carlo (MC) radiation transport package. The codes are coupled inmemory, allowing for the pin-by-pin fission source from the core simulator to be used by Shift. This unique capability allows the user to obtain both in-core and ex-core quantities by running a single simulation. Examples of ex-core calculations that can be performed include multicycle vessel fluence, detector response during reactor start-up and operation, and coupon fluence. One main advantage of the VERA ex-core capability is its flexibility and ease of use; users can select default settings with the standard VERA input for typical calculations or create their own ex-core geometry for specific cases. Also, Shift takes advantage of hybrid deterministic-MC methods to reduce variance and computational time. This paper details the full suite of VERA ex-core capabilities and provides input examples, simulation results, and computing resource use suggestions. These new capabilities have the potential to impact a wide user group in the nuclear community by enhancing and enabling high-fidelity light water reactor (LWR) ex-core calculations.
\end{abstract}

KEYWORDS: ex-core, CASL, hybrid methods

\section{INTRODUCTION}

The Virtual Environment for Reactor Applications (VERA) [1] created under the Consortium for Advanced Simulation of Light Water Reactors (CASL) enables pin-resolved radiation transport

\footnotetext{
*Notice: This manuscript has been authored by UT-Battelle, LLC, under contract DE-AC05-00OR22725 with the US Department of Energy (DOE). The US government retains and the publisher, by accepting the article for publication, acknowledges that the US government retains a nonexclusive, paid-up, irrevocable, worldwide license to publish or reproduce the published form of this manuscript, or allow others to do so, for US government purposes. DOE will provide public access to these results of federally sponsored research in accordance with the DOE Public Access Plan (http://energy.gov/downloads/doe-public-access-plan).
} 
coupled with thermal hydraulics, fuel pin heat transfer, nuclide transmutation, and corrosion chemistry. As large, high-fidelity reactor calculations become increasingly important in the context of lifetime extensions of the current nuclear fleet, CASL has expanded VERA's capabilities to include high-fidelity ex-core calculations. This was achieved by coupling VERA with the Shift Monte Carlo (MC) radiation transport package developed at Oak Ridge National Laboratory (ORNL) [2].

VERA's ex-core capability has been developed with a focus on flexibility and ease of use. Shift has been coupled with VERA in-memory, which allows data such as the pin-by-pin fission source calculated by VERA to be used by Shift and for Shift results to be accumulated and reported by VERA. Users can run with default settings with the standard VERA input for typical calculations or choose to create their own detailed ex-core geometry and MC tally regions for specific or atypical cases. Also, VERA takes advantage of the hybrid deterministic-MC methods available in Shift to reduce variance and computational time. Example applications include calculations of ex-core quantities for safety margins, reactor start-up analysis, and vessel lifetime.

This paper builds on previous work on coupon fluence calculations with VERA [3] by detailing the full suite of VERA ex-core capabilities and providing input examples, example simulation results, and computing resource use suggestions. Section 2 discusses the Shift coupling with VERA. Specific ex-core capabilities are reviewed in Section 3, and Section 4 demonstrates these capabilities with a model of Watts Bar Nuclear Plant Unit 1 (WBN1). Finally, Section 5 summarizes the VERA ex-core simulation capabilities and discusses future development.

\section{VERA-SHIFT COUPLING}

Shift is a massively parallel, continuous-energy (CE) MC radiation transport code available within VERA that employs a high-speed internal geometry package for light water reactors and solves neutron, photon, and coupled neutron-photon transport problems. Shift provides novel transport algorithms and hybrid methods tailored for leadership-class computing platforms, in addition to multiple geometries, tallies, and physics [2]. It has both fixed-source and eigenvalue solvers and can employ variance reduction via hybrid methods using the Denovo [4] deterministic transport code. A complete detailing of Shift as a stand-alone tool is available in [2,5].

By coupling Shift with VERA, detailed ex-core calculations can be conducted with minimal additional effort from the user. The basic coupling strategy between VERA and Shift involves transferring the fission neutron source generated by VERA to Shift in typical ex-core calculations, as depicted in Fig. 1; however, there is also an experimental capability to transfer detailed depleted isotopics, temperatures, and coolant densities. Shift then samples the fission source and runs a fixed-source calculation to determine the ex-core quantities of interest. Fixed-source transport can be run in neutron-only or coupled neutron-gamma modes. VERA and Shift run on different sets of processors; therefore, they can run different state points concurrently leading to faster run times.

\section{EX-CORE CAPABILITIES}

Coupling VERA with Shift has enabled several new capabilities for ex-core simulations with VERA, including CE particle transport, variance reduction with the Consistent Adjoint-Driven Importance Sampling (CADIS) method, flexible detailed ex-core geometry definition using the 


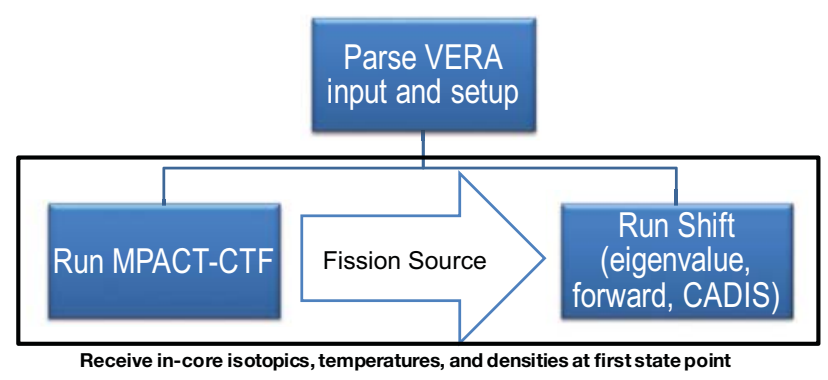

Figure 1: Diagram of Shift coupling with VERA for a single state point in a fuel cycle.

General Geometry (GG) package, and calculation of ex-core quantities with minimal user input. Details on specific ex-core capabilities are given in the following sections.

\subsection{Hybrid Variance Reduction}

Fixed-source MC simulations with Shift can be performed in either forward or CADIS [6] mode. Forward mode refers to a standard transport simulation without the use of advanced variance reduction techniques. In CADIS mode, the CADIS variance reduction method is used to reduce the computation time and statistical variance by optimizing transport for a quantity of interest. The implementation of the CADIS method is an important feature for VERA because computational resources can be a limiting factor in the use of CASL tools for increasingly large and complex problems. Without CADIS, it is extremely difficult to obtain particle interaction tallies in exvessel regions with high confidence using reasonable computational resources. To run in CADIS mode, only the problem mode parameter is required; however, many users will want to set other advanced parameters, and these details can be found in the VERA ex-core manual [7].

\subsection{Fission Source}

VERA sends Shift a pin-wise fission source distribution at each state point. There are three different options that can be used for the fission source spectrum in the Shift calculation: nuclide_watt, u235_watt, or mpact. The nuclide_watt option is the default and will sample the appropriate Watt spectrum for each of four nuclides: ${ }^{235} \mathrm{U},{ }^{238} \mathrm{U},{ }^{239} \mathrm{Pu}$, and ${ }^{\mathrm{Pu}} 241$. All neutrons are sampled from a ${ }^{235} \mathrm{U}$ Watt spectrum if the $u 235 \_w a t t$ option is used. If the mpact option is given, Shift will create a nonseparable source with the space-energy distribution calculated by VERA. In this case, the source energy distribution in Shift is determined by the energy group structure used for the eigenvalue calculation by VERA and may not be appropriate for ex-core applications. The fission source spectrum options and their impact on ex-core quantities are discussed in detail in [8].

\subsection{Detailed Ex-Core Modeling and Tallies}

One of the primary advantages of VERA for ex-core analyses is the ability to automatically set up a three-dimensional full-core model specified by the common VERA input. Thus, almost no additional user input is required to execute a Shift vessel fluence calculation for normal core follow 
analyses. This capability removes sources of error that can result from inconsistencies between the input models required by different codes. Using VERA to calculate the vessel fluence for simple ex-core geometries is straightforward; the user can specify a cylindrical mesh tally in the SHIFT block of the VERA input. During the ex-core simulation, Shift tallies the flux in the vessel and communicates it back to VERA for accumulation of the fluence over the entire cycle.

The common VERA input allows core pads and vessel radii to be specified; however, if the user requires a more detailed ex-core geometry, a supplemental ex-core file can be used to create an arbitrary geometry region outside of the core barrel using the GG package [3]. To facilitate this process, the ability to automatically generate the supplemental ex-core file via parameters defined in the standard VERA input has been implemented. The user can specify a bioshield parameter with materials in concentric cylinders beyond the vessel. Different detector types can also be defined and placed outside of the vessel. If additional details are wanted, the user can start from the auto-generated supplemental file. A current limitation of this capability is that materials used in the auto-generated file are taken from a template file and may not be consistent with the corresponding materials in the VERA common input. This discrepancy will be resolved in a future release.

Through the supplemental ex-core input file, the user has access to Shift's tally capabilities. Cell tallies can be specified for any cells defined in the supplemental input, and mesh tallies can be specified at any location in the geometry including the core. User-defined response functions can be used with these tallies (e.g., biological dose, detector response, material damage). Any cell tallies are communicated to VERA at each state point for writing to the VERA output file. Regardless of the level of detailed ex-core geometry a user decides is needed, only one simulation is required to calculate in-core and ex-core quantities of interest.

\subsection{Output and Postprocessing}

While Shift passes vessel flux and detector responses to VERA at each state point for inclusion in the VERA output file, an independent Shift HDF5 output file is also written. If requested in the SHIFT block, this file includes the fission source used and the results of all requested tallies. If running in CADIS mode, the adjoint function, pin importances, and weight windows can also be output to this file. A separate HDF5 file for each state point is output with material compositions as well as an HDF5 file with a raytrace of the geometry at each axial level requested in the VERA input file, which can be used for advanced problem geometry visualization. The results from a VERA ex-core simulation can also be visualized and postprocessed in the VERAView interactive graphical interface [9]. The ex-core quantities that can currently be analyzed include vessel flux, vessel fluence, and pin importances.

\section{VERA EX-CORE EXAMPLES}

The following examples use a model of cycle 1 of WBN1 [10] to demonstrate the VERA excore capabilities. The core is loaded with fresh fuel, and VERA is run with 32 state points. A detailed ex-core geometry was specified using a supplemental ex-core input file to define core pads, capsules and holders, vessel, insulation, bioshield, and source range and power range detectors. An automatically generated raytrace through the core midplane is shown in Fig. 2. Note that the incore geometry was still defined in the common VERA input up to the core barrel. 


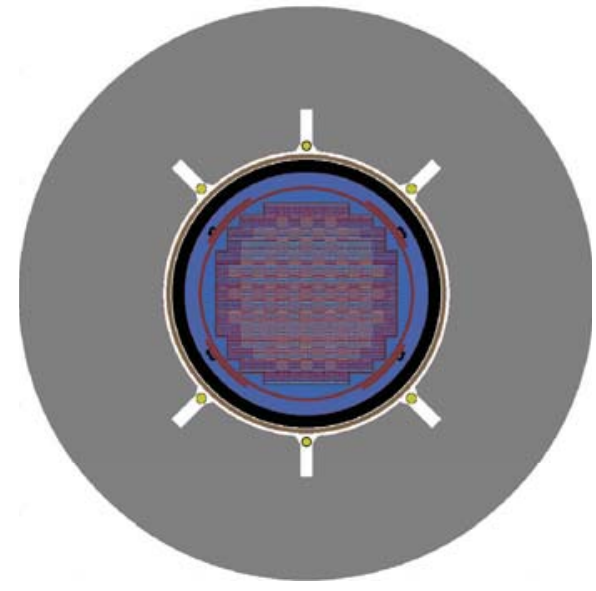

(a) Full geometry

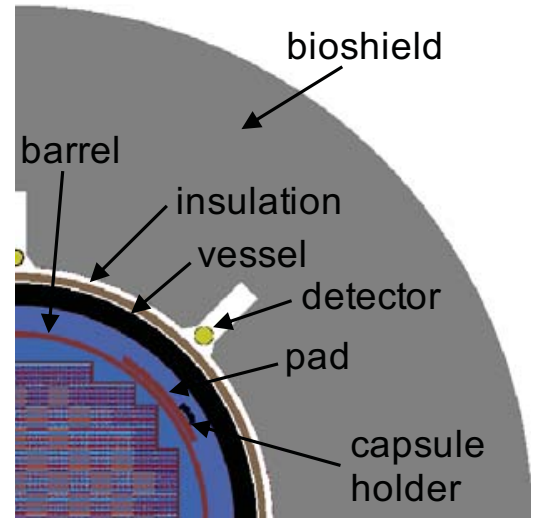

(b) Northeast quarter of geometry

Figure 2: Raytrace of the WBN1 geometry at the core midplane $(z=200 \mathrm{~cm})$.

As discussed in Section 3.3, a supplemental ex-core file for modeling details beyond the reactor pressure vessel can be automatically generated from the VERA common input file by using the bioshield, det, and det locations parameters in the CORE block. An example of these parameters for the WBN1 model is given in Listing 1. The raytrace generated when running VERA with this input is shown in Fig. 3. This example defines two ex-core detector types: a power range detector identified as $P W R$ and a source range detector identified as $S R C$. Note that both detector types use a wedge-shaped well in the bioshield. Four power range detectors and two source range detectors are placed using the det_locations parameter.

By defining mesh tallies in the supplemental ex-core input file, the flux in the baffle was determined. The baffle tally result for state point 3 of WBN1 cycle 1 is given in Fig. 4. This demonstrates that while the supplemental ex-core input file defines only the geometry beyond the barrel, mesh tallies can be defined in any part of the geometry. After completing the simulation, the pin importances, pin powers, and accumulated vessel fluence were visualized with VERAView, as shown in Fig. 5. This simulation was run in CADIS mode and optimized transport for the vessel flux tally. In Fig. 5a, the plotted pin importances, defined as the importance of each pin to the vessel flux, show that only the outermost pins contribute significantly to the vessel flux as expected.

An example of computing resources used for a simple WBN1 vessel fluence calculation are given in Table 1. These calculations were performed on an ORNL institutional cluster with $128 \mathrm{~GB}$ memory nodes consisting of 32 Intel Xeon v3 CPUs and InfiniBand node interconnects. The same number of particle histories were simulated in Shift for the CADIS and forward calculations. The time presented per state is the CPU time per code to perform the solve as averaged over all states and the total CPU time is the actual total execution time. The improvement in FOM seen when using CADIS for this example Shift vessel fluence calculation ranged from 55 to 80 per state. 


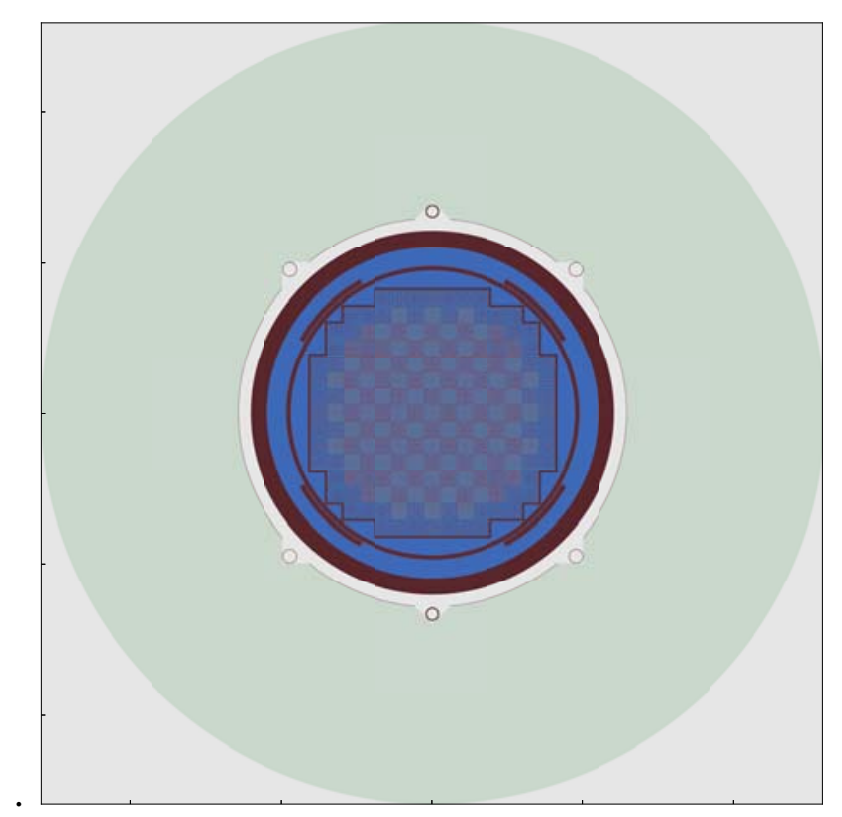

Figure 3: Raytrace through an automatically generated ex-core geometry for WBN1.

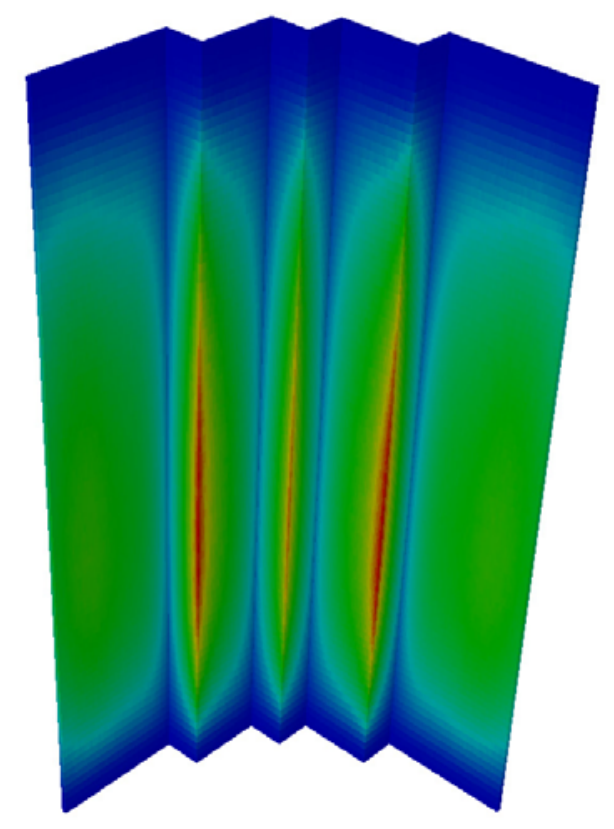

Figure 4: Visualization of baffle tally results in the northeast quadrant of WBN1.

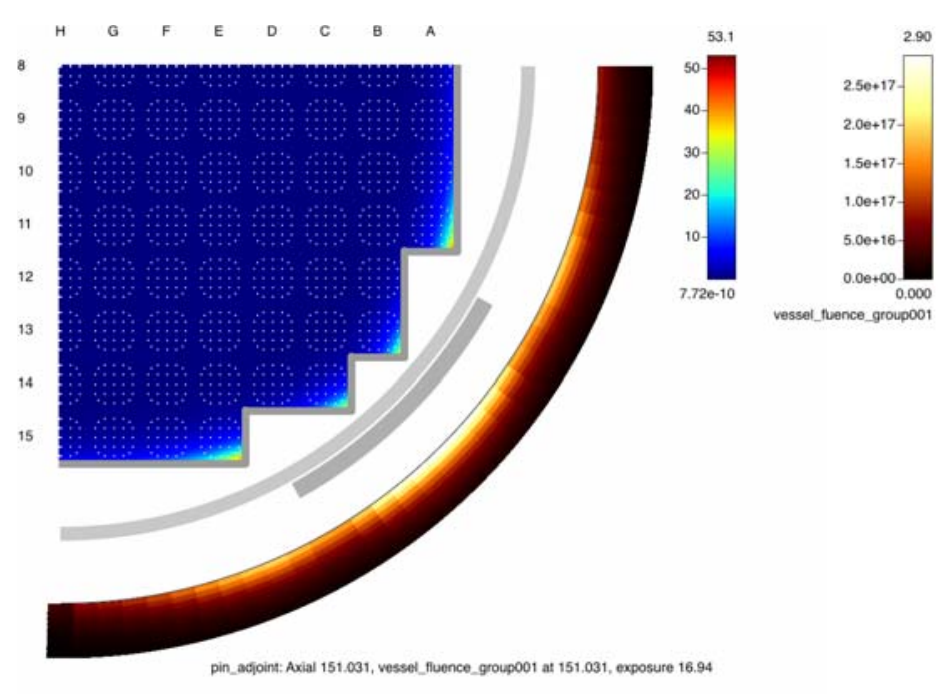

(a) Pin importances in-core and vessel fluence ex-core

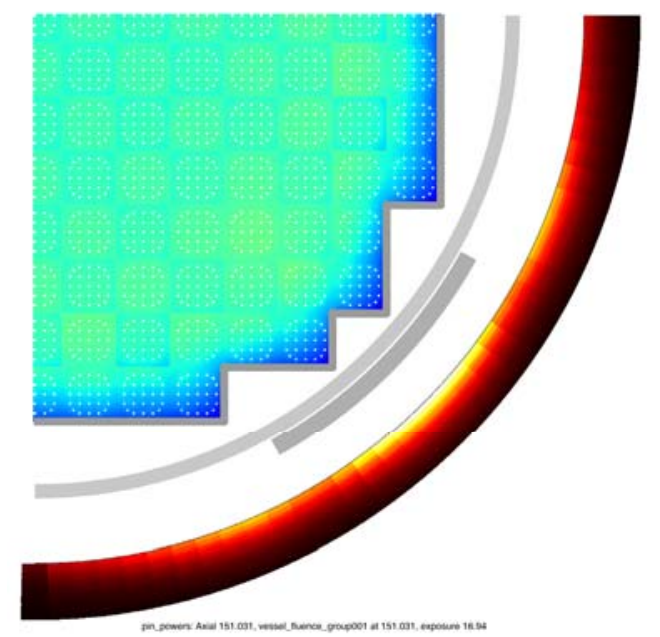

(b) Normalized pin power in-core and vessel fluence ex-core

Figure 5: Example of visualizing the results of a VERA ex-core simulation of WBN1 with VERAView. 
Table 1: VERA computing resources for WBN1 vessel fluence calculations.

\begin{tabular}{lccc}
\hline Code & Processors & $\begin{array}{c}\text { CADIS Timing } \\
\text { (avg. per state) }\end{array}$ & $\begin{array}{c}\text { Forward Timing } \\
\text { (avg. per state) }\end{array}$ \\
\hline MPACT-CTF & 928 & $45 \mathrm{~min}$ & $45 \mathrm{~min}$ \\
Shift & 400 & $66 \mathrm{~min}$ & $38 \mathrm{~min}$ \\
\hline Total (32 states) & 1328 & $2,231 \mathrm{~min}$ & $1,641 \mathrm{~min}$ \\
\hline
\end{tabular}

\section{CONCLUSIONS}

The capabilities of VERA have been expanded to perform high-fidelity ex-core calculations by coupling with the Shift MC code. This enables detailed modeling of the ex-core geometry for the determination of ex-core quantities, including vessel fluence, coupon fluence, and detector response. New parameters in the common VERA input allow for the user to perform ex-core calculations with minimal additional effort. These new capabilities can enable a wide user group in the nuclear community to perform high-fidelity ex-core calculations.

Several additional features are either in progress or planned to further expand VERA's ex-core capabilities. Some of these will enable VERA to make use of features that are already available in Shift, including domain decomposition and Forward Weighted CADIS (FW-CADIS). Domain decomposition is needed to run full-core calculations with fully coupled isotopics, temperatures, and densities sent to Shift. FW-CADIS will be important for calculations focused on regions above the active fuel and with multiple detectors in different regions. Other future features include using sources besides the fission source and updating variance reduction parameters at select state points.

\section{ACKNOWLEDGMENTS}

This research was supported by the Consortium for Advanced Simulation of Light Water Reactors (http://www.casl.gov), an Energy Innovation Hub (http://www.energy.gov/hubs) for Modeling and Simulation of Nuclear Reactors under US Department of Energy (DOE) Contract No. DE-AC0500OR22725. This research also used resources of the Compute and Data Environment for Science (CADES) at ORNL, which is supported by the DOE Office of Science.

\section{REFERENCES}

[1] J. A. Turner, K. Clarno, M. Sieger, R. Bartlett, B. Collins, R. Pawlowski, R. Schmidt, and R. Summers. "The Virtual Environment for Reactor Applications (VERA): Design and architecture." Journal of Computational Physics, volume 326, pp. 544-568 (2016).

[2] T. M. Pandya, S. R. Johnson, T. M. Evans, G. G. Davidson, S. P. Hamilton, and A. T. Godfrey. "Implementation, capabilities, and benchmarking of Shift, a massively parallel Monte Carlo radiation transport code." Journal of Computational Physics, volume 308, pp. 239-272 (2016). 
[3] E. E. Davidson, T. M. Pandya, A. T. Godfrey, and M. Asgari. "Watts Bar I Ex-Core Analyses Using VERA." In $20^{\text {th }}$ Topical Meeting of the Radiation Protection Shielding Division. American Nuclear Society (2018).

[4] T. Evans, A. Stafford, R. Slaybaugh, and K. Clarno. "Denovo-A new three-dimensional parallel discrete ordinates code in SCALE." Nuclear Technology, volume 171, pp. 171-200 (2010).

[5] S. R. Johnson, T. M. Evans, G. G. Davidson, S. P. Hamilton, and T. M. Pandya. "Exnihilo Transport Code Manual." Technical Report Release 6.2.0 (Dev), Oak Ridge National Laboratory (2017).

[6] J. C. Wagner and A. Haghighat. "Automated variance reduction of Monte Carlo shielding calculations using the discrete ordinates adjoint function." Nuclear Science and Engineering, volume 128(2), pp. 186-208 (1998).

[7] T. Pandya, T. Evans, K. Royston, K. Clarno, and B. Collins. "Excore Radiation Transport Modeling with VERA: Manual." Technical Report CASL-U-2018-1556-001, CASL (2018).

[8] E. E. Davidson, T. M. Pandya, K. E. Royston, T. M. Evans, A. T. Godfrey, and S. C. Henderson. "Effect of Fission Source Spectrum on Monte Carlo Calculation of Ex-core Quantities." In Proceedings of the PHYSOR 2020. American Nuclear Society (2020).

[9] A. T. Godfrey and R. W. Lee. "VERAView User's Guide." Technical Report CASL-U-20161058-000, CASL (2016).

[10] J. C. Gehin, A. T. Godfrey, F. Franceschini, T. M. Evans, B. Collins, and S. Hamilton. "Operational Reactor Model Demonstration with VERA: Watts Bar Unit 1 Cycle 1 Zero Power Physics Tests." Technical Report CASL-U-2013-0105-001, CASL (2013).

\section{APPENDIX A. Automated Detector Input}

\section{Listing 1: Watts Bar Unit 1 input example with automated excore detectors.}

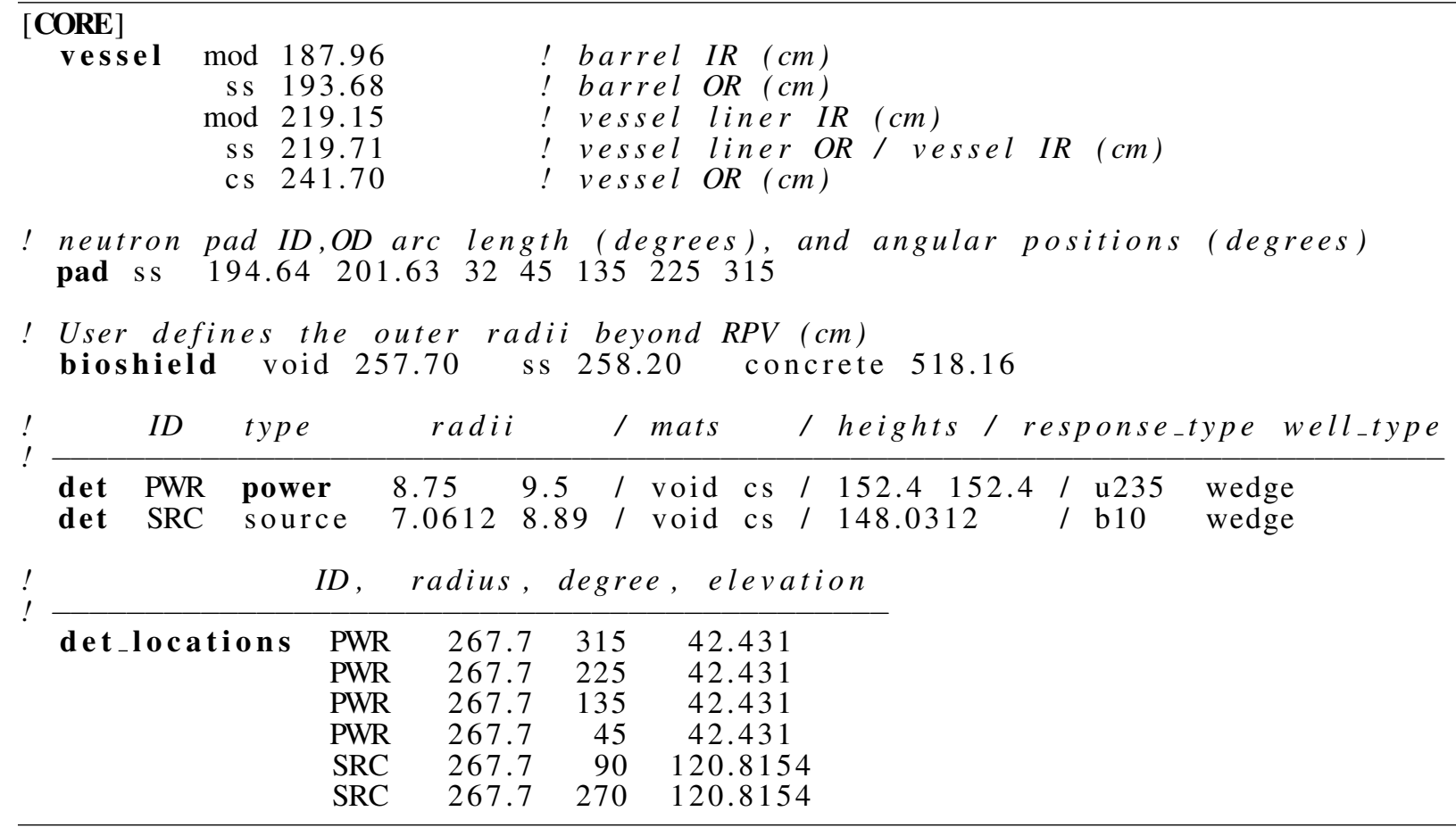

